\title{
Predictive Scheduling in Multi-Carrier Wireless Networks with Link Adaptation
}

\author{
Gokhan Sahin \\ Department of Computer Science and Engineering \\ University of Nebraska-Lincoln, \\ Lincoln, Nebraska \\ Email: gsahin@cse.unl.edu
}

\author{
Fanchun Jin, Amrinder Arora, Hyeong-Ah Choi \\ Department of Compuer Science \\ The George Washington University \\ Washington, DC \\ Email: \{jinfc,amrinder,hchoi\}@gwu.edu.
}

\begin{abstract}
Channel-aware scheduling and link adaptation methods are widely considered to be crucial for realizing high data rates in wireless networks. However, predicting the future channel states, and adjusting the transmission schedules and parameters accordingly, may consume valuable system resources, such as bandwidth, time, and power. This paper considers the trade-offs between the prediction quality and the throughput in a wireless network that uses link adaptation and channel-aware scheduling. In particular, we study the effects of the look-ahead window, i.e., the range of future time slots on which we have channel state estimates, and the reliability of the channel state estimates on the throughput. We develop an online scheduling algorithm for a multi-channel multi-user network that employs predictive link adaptation, and generalize it to incorporate imperfect channel state estimates. We use this heuristic together with performance bounds to the offline version of the problem to evaluate the performance with varying prediction quality. Our results suggest that it may be possible to reap most of the potential channel-aware scheduling benefits with a small look-ahead, and imperfect channel state estimates. Thus, a modest consumption of resources for channel prediction and link adaptation may result in a significant throughput improvement, with only marginal gains through further enhancement of the prediction quality. Our results can provide meaningful guidelines in deciding what level of system resource consumption is justified for channel quality estimation and link adaptation.
\end{abstract}

\section{Motivation}

This paper considers the downlink scheduling problem in a multi-carrier wireless network that uses link adaptation. Link Adaptation (LA), which loosely refers to changing transmission parameters over a link, such as modulation, coding rate, power, etc., in response to changing channel conditions is considered to be a powerful means of achieving higher efficiency or throughput in wireless networks. The adaptation of the transmission parameters is performed according to the predicted future quality of the channel, also called as the channel state (CS).

While it is desirable to adapt the transmission parameters according to the channel state information (CSI) to capture even small-scale variations, there are practical limitations to channel state prediction and link adaptation. Frequent adaptation increases the number of mode-change messages transmitted over the channel, consuming bandwidth, and time resources [1]. While many aspects of scheduling transmissions over time-varying wireless channels have been studied (see, for example, [2], [3], [4] and the references therein), the penalty induced by LA has not been considered. Moreover, predicting the future channel quality may also consume significant amount of system resources (e.g., time, bandwidth and power), since it may involve transmission of training-sequences, pilot tones, or feedback messages carrying the CSI. Naturally, the additional cost and complication of predicting CS and LA increase with the desired prediction accuracy. The contributions of this paper are two-fold. Firstly, we explicitly consider the time-penalty induced by the transmission-mode changes in the scheduling problem, and develop an online multi-user, multichannel scheduling algorithm for this problem. Secondly, we study the trade-offs between the channel-state prediction quality and the throughput. In particular, we consider two different metrics of prediction quality: (i) Look-ahead, defined as the number of future time slots over which CSI is available at the decision time for the current time slot, and (ii) prediction error, which is the probability that the estimate for a future channel state is different from the actual one. The results we obtain can provide meaningful guidelines in deciding what level of system resource consumption (bandwidth, time, power, and additional complexity) is justified for channel quality estimation and link adaptation.

The rest of the paper is organized as follows. In Section II, we discuss link adaptation techniques in general. In Section III, we present the predictive scheduling problem for a specific system that uses closed-loop LA, and describe an online scheduling algorithm for this problem in Section IV. The algorithm is extended to incorporate errors in CS prediction in Section V. We evaluate the performance of the algorithms, and the trade-offs between prediction quality and throughput in Section VI. Section VII concludes the paper.

\section{Link AdAptation Techniques}

Link Adaptation attempts to exploit the variations in the quality of the wireless channel by modifying a set of transmission parameters, such as the modulation, and coding modes. This is suitable for duplex communication, since the transmission parameters have to be adapted using some form of twoway transmission [5]. For simplicity, let us consider the link adaptation for downlink transmission of data over a channel from a base-station to a mobile terminal. In this context, 
the adaptation of the transmission parameters is done by the transmitter at the base station.

There are three components of link adaptation [5], [1]:

- Estimation of the Channel Quality: In order to adjust the transmission parameters for the next transmission, a reliable estimate of the channel quality for the next transmission time-slot(s) is needed.

- Transmission Parameter Selection: Based on the estimate of the channel quality for the next, and possibly subsequent, time-slot(s), the best transmission parameters are selected.

- Signaling (or blind detection) of the selected parameters: The selected transmission parameters also need to be known by the receiver in order to decode/receive the transmitted packet. Depending on the system, this information can be either explicitly signaled to the receiver, or detected blindly by the receiver.

The actual mechanisms used to implement the three components of LA are system-dependent, and affect the overall performance. The quality of the channel for the future transmission slots can be obtained based on the past channel quality estimations or measurements. Depending on whether feedback is used or not, LA methods can be classified as open-loop adaptation and closed-loop adaptation [5]. In openloop adaptation, the transmission parameters are selected by the transmitter without any feedback from the receiver. This method could be useful, for example, in a reciprocal channel, where the communication is bidirectional and both uplink and downlink can be assumed to have the same channel quality. Thus, each station can estimate the channel quality on the basis of its received signal. In closed-loop adaptation, the channel quality experienced in two directions may not be symmetrical, and therefore the receiver has to signal its perceived channel quality explicitly to the transmitter. This method would be required in any non-reciprocal channel, or in the presence of interference.

Many metrics, also called channel state information (CSI) can be used for estimating the channel quality. Signal-to-noiseratio (SNR), or signal-to-noise-plus-interference-ratio (SINR) may be available from the physical layer. This can be done, for example, by sending training sequences which do not carry data, or by sending pilot tones to obtain an estimate of the channel transfer function [5]. At the link layer, packet error rates (PERs) may be available. This requires, updating and maintaining data reception history for each mode until all modes have been trained and appropriate PER thresholds for each mode have been obtained. A reliable estimate of PER may require up to thousands of packets for a given mode, resulting in a slow adaptation loop [1].

Once estimates of the channel state are obtained, one or more of the transmission parameters can be adjusted. These parameters may include modulation type, several coding parameters (code rate, adaptive interleaving and puncturing for convolutional and turbo codes, and block lengths for block codes), and transmission power. When these parameters are modified on one end of the communication link, the other end needs to be notified of the new parameters to ensure proper reception of the future symbols/packets.

Clearly, LA may consume significant amount of system resources (bandwidth, time, power) depending on the frequency of the adaptation, and the quality of the channel state estimation required. If, for example, the CSI is estimated based on transmission of training sequences, then the duration and frequency of sending these training sequences may need to be increased. In this paper, we are interested in the effects of the prediction quality on the scheduling problem in a wireless network with link adaptation. The results we obtain may be useful in evaluating various LA and channel state prediction techniques in terms of their value from the throughput point of view.

\section{Problem Statement}

Multi-User Multi-Channel Scheduling with Closed-loop Link Adaptation: We consider the downlink scheduling problem in a wireless network with $N$ mobile stations (MS) and $F$ channels (sub-carriers). Time is slotted, and time-divisionduplexing is used, i.e., uplink and downlink transmissions take place in turn. The base-station (BS) may experience a different time-dependent channel quality to each mobile station (MS) on each sub-carrier. The actual transmission data rate towards user $n$ in a time slot $t$ on channel $f$ may take one of $M$ discrete values, but is upper bounded by the corresponding capacity $c_{n, f}(t)$ which represents the channel state. The data rate is adjusted using closed-loop adaptation: the MSs (receivers) estimate the CSI based on the previous downlink transmission(s), and pass this information to the BS during the uplink transmission. At the beginning of time slot $i$, BS has perfect knowledge of the CS in time slot $i$, as well as imperfect estimates of the CS over time slots $i+1, i+2, \ldots i+K$, where $K$ is the look-ahead.

At the beginning of each time slot $i$, for each sub-carrier, the BS decides to which MS, and at what data rate, it is going to transmit during time slot $i$. If a change is required in the receiving $\mathrm{MS}$ or the data rate (by varying the modulation rate, coding rate, etc.) for a sub-carrier $f$, then the next downlink transmission (in time-slot $i$, sub-carrier $f$ ) is used to advertise the new user/rate of the channel, which may be confirmed by the MS through an ACK in the next uplink transmission. Thus, transmission at the new data rate (possibly to a new user) starts only after a delay of a full duplex transmission cycle. If the $\mathrm{BS}$ decides to change the transmission rate (or the MS) at the beginning of time slot $i$, then transmission with the new rate starts at the beginning of time slot $(i+1)$.

Let $c_{n, f, i}^{\prime}(j)$, where $K+i \geq j>i$ be the estimate available in time slot $i$ for $c_{n, f}(j)$. Let $P_{n, f, i, j}(a, b)$ be the probability that the actual $c_{n, f}(j)=b$, given that $c_{n, f, i}^{\prime}(j)=a$, and let $x_{n, f}(i)$ denote the selected transmission rate for user $n$ in time slot $i$ on sub-carrier $f$. At the beginning of each time slot $i$, BS has the knowledge of $x_{n, f}(i-1), c_{n, f}(i), c_{n, f, i}^{\prime}(j)$, and $P_{n, f, i, j}(a, b)$ for each user $n$ and each channel $f$. Given this information, it has to choose $x_{n, f}(i), x_{n, f}(i) \leq c_{n, f}(i)$. The goal is to maximize $\sum_{n=1}^{N} \sum_{f=1}^{F} \sum_{t=1}^{T} x_{n, f}(t)$, where $T$ denotes the overall transmission period. The decision has 
to satisfy the following system constraints: 1. Each channel can be used for transmission to only one MS at a given time slot; 2. The data transmission rate has to be selected from one of the $M$ discrete transmission rates available (but less than the time and user dependent capacity); 3. On each channel, changing the data rate, or the intended MS, makes the next time slot useless in terms of data transmission; 4. Each MS $n$ can use at most $\alpha(n)$ channels in a time-slot.

\section{Predictive Multi-User Multi-Channel SCHEDULING ALGORITHM}

Predictive Multi-User Multi-Channel (PMUMC) scheduling algorithm is an online scheduling algorithm that attempts to optimize the throughput by making use of the knowledge of the CS for all users and channels in the current time slot $\left(c_{n, f}(i)\right)$, as well as the predicted CS for the following time slot (denoted as $c_{n, f}^{\prime}(i+1)$ from now on). We have restricted the heuristics in this paper to at most one lookahead, since, as we shall elaborate in subsequent sections, a single look-ahead yields most of the potential benefits of future CS prediction in our experiments, even when the estimates are imperfect.

PMUMC PSEUDO-CODE for time slot $i$

Given: (i) $a(f, i-1)$, and $x_{n, f}(i-1) \forall n, f$ : the user assigned to each channel, and the transmission rates for each channel and user in the previous time slot,

(ii) $c_{n, f}(i), c_{n, f}^{\prime}(i+1) \forall n, f$ : the capacity in the current time slot, and the estimated capacity in the next time slot

Find: $a(f, i)$ and $x_{n, f}(i), \forall n, f:$ the user assigned to each channel, and the transmission rates for each channel and user in the current time slot.

/ / Initially, no channel is assigned to any user

1 Set_a $a(f, i)=$ NULL $\forall f$

2 Set_ $A_{n}(i)=0 \forall n$

/ / Assign users and rates to each channel sequentially based on the optimal tentative two-slot schedule.

3 for (each channel $f$ ) \{

4 Compute $\left\{\forall n: x_{n, f}(i), x_{n, f}^{\prime}(i+1)\right\}$ using MUWDH

$5 \quad$ Set $a(f, i)=\arg \max _{n: A_{n}(i)<\alpha(n)}\left\{x_{n, f}(i)+x_{n, f}^{\prime}(i+\right.$ 1)\}

$6 \quad$ Set $A_{a(f, i)}(i)=A_{a(f, i)}(i)+1$

$7 \quad$ Set $x_{n, f}(i)=0 \forall n \neq A_{a(f, i)}(i)$ $8\}$

Fig. 1. Predictive MUMC scheduling algorithm.

PMUMC, whose pseudo-code is given in Figure 1, is an online algorithm which uses three consecutive time-slots as a building block in a sliding-window fashion: the transmission decisions for slot $i$ are determined based on the transmission decisions for slot $i-1$, and capacity parameter inputs for slots $i$ and $i+1$. Define the assignee $a(f, i)$ as the user (if there is one) who is granted transmission on channel $f$ in slot $i$. Let $x_{n, f}(i)$ be the transmission rate used by MS $n$ on channel $f$ in slot $i$, and $A_{n}(i)$ be the total number of channels assigned to user $n$ in time slot $i$. By definition, $x_{n, f}(i)>0$ only for $n=a(f, i)$. At the beginning of time slot $i$, PMUMC selects $x_{n, f}(i)$, and hence $a(f, i)(\forall n, f)$ as follows. Given the assignees and transmission rates assigned to the channels in the previous slot $\left\{a(f, i-1), x_{n, f}(i-1): \forall n, f\right\}$, and the channel states in the current and next time slots, $\left\{c_{n, f}(i), c_{n, f}^{\prime}(i+1): \forall n, f\right\}$, it assigns users and transmission rates for slot $i$ to the channels sequentially. For each channel, the assignee is selected as the user that would maximize the transmission over the two time-slot period $(i, i+1)$ while meeting the constraint on the number of channels that can be assigned to it. For a given channel $f$, PMUMC utilizes multi-user wait-dominate-hold (MUWDH), which computes for each user $n$ a tentative twoslot schedule $x_{n, f}(i), x_{n, f}^{\prime}(i+1)$ that maximizes the tentative throughput $x_{n, f}(i)+x_{n, f}^{\prime}(i+1)$, assuming that user $n$ is granted transmission in both slots on channel $f$. PMUMC then selects the MS with the highest tentative throughput while not exceeding the channel count limit as $a(f, i)$, the actual user for channel $f$ in slot $i$, with the corresponding transmission rate $x_{n, f}(i)$. We emphasize that $x_{n, f}^{\prime}(i+1)$ values computed in slot $i$ are only projections, and they may not materialize later. This is because similar computations will be performed in slot $i+1$ with one slot shift in time.

We next describe MUWDH, a generalized version of the single-channel single-user algorithm in [6]. For a given channel $f$, let $m_{n, f}(i)$ denote the maximum usage achievable in the current slot by user $n$, given $a(f, i-1), x_{n, f}(i-1)$ and $c_{n, f}(i)$. Then, we have three different cases. (I) $m_{n, f}(i)=0$ if $n=a(f, i-1)$ and $c_{n, f}(i)<x_{n, f}(i-1),($ II $) m_{n, f}(i)=$ $c_{n, f}(i)$ if $a(f, i-1)=$ NULL, and $(I I I) m_{n, f}(i)=x_{n, f}(i-1)$ if $n=a(f, i-1)$ and $c_{n, f}(i) \geq x_{n, f}(i-1)$. Then, the tentative schedule for each user and channel is constructed by choosing one the following three actions.

(i) Wait: If the (estimated) next capacity is more than twice the maximum possible current usage, set the current usage to 0 (so that the next capacity can be used fully).

(ii) Dominate: If the maximum possible current usage is more than twice the next capacity, set the current usage to maximum possible current usage. Usage for the next time slot will be 0 . (iii) Hold: If neither of the above is the case, use the minimum of the two capacities if the channel was not used by any user in the previous slot (so that both the current and next time slot may be potentially used). If the user was already using the channel in the previous time slot, then use the maximum possible current usage.

The precise set of rules for choosing $x_{n, f}(i)$, and $x_{n, f}^{\prime}(i+1)$ are as follows.

(i) if $c_{n, f}^{\prime}(i+1)>2 m_{n, f}(i)$, set $x_{n, f}(i)=0$, $x_{n, f}^{\prime}(i+1)=c_{n, f}^{\prime}(i+1)$

(ii) if $2 c_{n, f}^{\prime}(i+1)<m_{n, f}(i)$, set $x_{n, f}(i)=m_{n, f}(i)$, $x_{n, f}^{\prime}(i+1)=0$

(iii) if $1 / 2 c_{n, f}^{\prime}(i+1) \leq m_{n, f}(i) \leq 2 c_{n, f}^{\prime}(i+1)$, and $x_{n, f}(i-1)>0$ set $x_{n, f}(i)=m_{n, f}(i)$. Set $x_{n, f}^{\prime}(i+1)=0$ if $m_{n, f}(i)>c_{n, f}^{\prime}(i+1)$, and $x_{n, f}^{\prime}(i+1)=m_{n, f}(i)$ otherwise 
(iv) if $1 / 2 c_{n, f}^{\prime}(i+1) \leq m_{n, f}(i) \leq 2 c_{n, f}^{\prime}(i+1)$, and $a(f, i-1)=$ NULL, set $x_{n, f}(i)=x_{n, f}^{\prime}(i+1)=$ $\min \left(m_{n, f}(i), c_{n, f}^{\prime}(i+1)\right)$.

\section{SCheduling With IMPERfEct Estimates AND Projections: PMUMC-PROJECTION CORRECTION}

Recall that PMUMC uses a tentative two slot schedule in assigning the users and the transmission rates to the channels. While the transmission in the current time slot $i$ is immediately materialized with rate $x_{n, f}(i)$ once the decision is made, the projected transmission for the next time slot $x_{n, f}^{\prime}(i+1)$ may not materialize for two reasons. First, the capacity estimations $c^{\prime}$ for slot $i+1$ may be imperfect, and thus the schedule may need to be modified in slot $i+1$. Secondly, note that PMUMC is a one-look-ahead algorithm, and its decisions are based on sliding windows of three time-slots. The actual transmission schedule for slot $i+1$ will be made in slot $i+1$, depending on the capacity estimates of slot $i+2$, and thus may be very different from the projections made for $x_{n, f}^{\prime}(i+1)$ in slot $i$.

In order to reflect the fact that the projected $x_{n, f}^{\prime}(i+1)$ may or may not materialize, we propose PMUMC with Projection Correction (PMUMC-PC). PMUMC-PC still selects the user for each channel based on the tentative schedules $x_{n, f}(i), x^{\prime} n, f(i+1)$. However, it gives higher importance to $x_{n, f}(i)$ by modifying the step 5 of the PMUMC in Figure 1 as follows:

5' Set $a(f, i)=\arg \max _{n: A_{n}(i)<\alpha(n)}\left\{x_{n, f}(i)+W x_{n, f}^{\prime}(i+\right.$ 1) $\}$,

where $0 \leq W \leq 1$ is a parameter that reflects the relative importance of the next transmission. Intuitively, inaccuracies in the future CS estimates would favor a smaller $W$. Similarly, the diversity due to presence of multiple users and channels would also favor a smaller $W$, since it introduces many more parameters that could cause the projected $x_{n, f}^{\prime}(i+1)$ not to materialize in slot $i+1$. These factors will be discussed further in our numerical results.

\section{NUMERICAL RESULTS}

In this section, we evaluate the performances of PMUMC and PMUMC-PC, and quantify the effects of the channel prediction quality on the system throughput. We present the throughput with varying levels of prediction reliability for three algorithms: scheduling with no look-ahead, scheduling with single look-ahead with various levels of projection correction (PMUMC-PC), and scheduling with single look-ahead (PMUMC). Note that PMUMC-PC subsumes the zero lookahead (when $W$ is set to 0), and PMUMC (when $W$ is set to 1).

In order to generate the channel state estimates $\left(c_{n, f}^{\prime}(i+1)\right)$ in our experiments, we assume that the channel estimate can take values from 9 data rates (in kb/s: 8.8, 11.2, 14.8, 17.6, $22.4,29.6,44.8,54.5,59.2)$ given in [1] with equal probability. The estimated channel states were altered according to the following estimation error model in order to obtain the actual channel states. $P_{n, f, i, i+1}(a, b)=p$ if $a=b$, and
$P_{n, f, i, i+1}(a, b)=(1-p) /(M-1)$, otherwise. We will refer to $p$ as the prediction accuracy. The duration of each experiment was 1000 time slots, and each data point shown is an average over 5 experiments.

Performance Bounds: The throughput achieved by the algorithms for each case is evaluated with respect to its ratio to an upper bound to the throughput obtained using the off-line version of the problem. For the multi-user single channel case, the upper bound is the optimal solution obtained to the off-line version of the problem. For multi-channel multi-user systems, we obtain two different bounds, and take the minimum of the two. The first one, is obtained simply by adding the multiuser single-channel bound over all channels. Note that this is an upper bound (even for the off-line case), since it does not constrain the number of channels that can be simultaneously assigned to a MS. The second upper bound is obtained by solving the offline problem without taking the one-slot link adaptation penalty into account. The algorithmic details of these bounds can be found in [7].

Observations: Figures 2-7 show throughput performances for systems with different number of users $(\mathrm{N})$, channels $(\mathrm{F})$, and channel count limit $(\alpha)$. We make the following observations.

1. The Effect of Look-ahead: Recall that PMUMC-PC with $W=0$ corresponds to a zero look-ahead algorithm, whereas all other cases correspond to single look-ahead, since they consider the projected transmission in the next time slot while deciding the schedule for the current slot. Figures 2-7 show that using the look-ahead information results in significant performance improvements in all cases. For a single-channel single-user system (Fig. 2), PMUMC ( $W=1)$ results in a throughput of $97 \%$ when the estimates are completely reliable, whereas the throughput with zero look-ahead is only $43 \%$. Even when the capacity estimates for the next slot are only $50 \%$ accurate, PMUMC achieves $86 \%$ of the optimal throughput that can be achieved by the offline algorithm. Similarly, the throughput achievable by PMUMC-PC, assuming perfectly accurate prediction of the capacities in the next slot, is about $95 \%$ in a single-channel system with 4 users (Fig. 3), 85\% in a 5-user 5-channel system (Fig. 4), and about $95 \%$ in the cases of a 20-user 5-channel system (Fig. 5), and a 40-user 5-channel system (Fig. 6). Thus, significant throughput improvements can be achieved by predicting the capacities in the next time-slot, which may justify the resource consumption and complexity in obtaining these predictions. It is also important to note that the throughputs achieved by single look-ahead algorithms are not only better than zero look-ahead, but also fairly high compared with the upper bounds. This may suggest that there is limited scope for further performance improvements through incorporating longer lookahead horizons.

2. The Effect of Imperfect Estimates: Figures 2-7 also show how the throughput changes as the prediction accuracy $(p)$ for the next slot capacities varies between 0.5 and 1. As expected, there is a consistent degradation in the performance of PMUMC-PC with increasing prediction inaccuracies for a fixed value of $W$. For example, for the 4-user 1-channel 
system of Fig. 3, PMUMC-PC with $W=0.7$ achieves a throughput of $89 \%$ when prediction accuracy is 0.5 , whereas it can achieve $94 \%$ when accuracy is 1 . However, we note that PMUMC-PC can still give better performance than a zero look-ahead algorithm in all our experiments, even in the presence of significant prediction inaccuracies. This suggests that it may not be necessary to obtain $100 \%$ reliable estimates in order to take advantage of a look-ahead, especially if a sacrifice in estimation accuracy could result in savings in the consumption of resources for prediction. It is also interesting to note that the optimal value of the weighting parameter $W$ for PMUMC-PC tends to decrease with decreasing estimation accuracy. This is expected, since the relative importance of the projected transmissions over the next slot should be reduced when the estimates are not accurate.

3. The Effect of Multi-User Diversity: As discussed in Section V, the projected schedule for the next time slot computed by PMUMC-PC may not be realized even when the predictions are accurate, since the decision at each time slot considers only a single look-ahead: new information about the capacities in time slot $i+2$ becomes available only in slot $i+1$, possibly changing the projected schedule. Presence of multiple users and channels introduces even more parameters that can change the projected schedule, thus favoring a lower weight $W<1$ for the PMUMC-PC algorithm. The multiuser diversity effect can be observed in Figures 4-6 for a 5channel system with varying number of users. The optimal $W$ for PMUMC-PC decreases from $W=0.7$ to $W=0.1$ as $N$ increases from 5 to 40 . Multi-user diversity, indeed favors a smaller $W$, and hence a greedier algorithm that focuses on the current slot, rather than the next slot in this case. This diversity effect can also be observed when comparing a 4-user singlechannel system (Figure 3) with a single-user single-channel system (Figure 2). The optimal value of $W$ for the 4-user system is always less than 1 , while single-user system is quite insensitive to $W>0$.

4. The Effect of Allowing Multiple-Channels per User: Depending on the system constraints, each user may be allowed to utilize more than one channel in a given time slot. Figure 7 shows the throughput performance in a 5-channel 5 user system with $\alpha=4$. We note that PMUMC-PC achieves a high throughput for this case as well, reaching up to $94 \%$. We also note that this throughput is considerably higher than the corresponding system with $\alpha=1$, where throughput reaches only up to $85 \%$ (Fig. 4). Thus, for this particular case, allowing a MS to use multiple channels simultaneously can lead to about $10 \%$ performance improvement.

\section{CONCLUSION}

Channel-aware scheduling and link adaptation will play an important role in achieving the high data rates required by emerging fixed and mobile applications. In this paper, we have focussed on the effects of the prediction quality on the system throughput for downlink transmission scheduling. We have developed a multi-user multi-channel single lookahead scheduling algorithm (PMUMC-PC), which can incor-

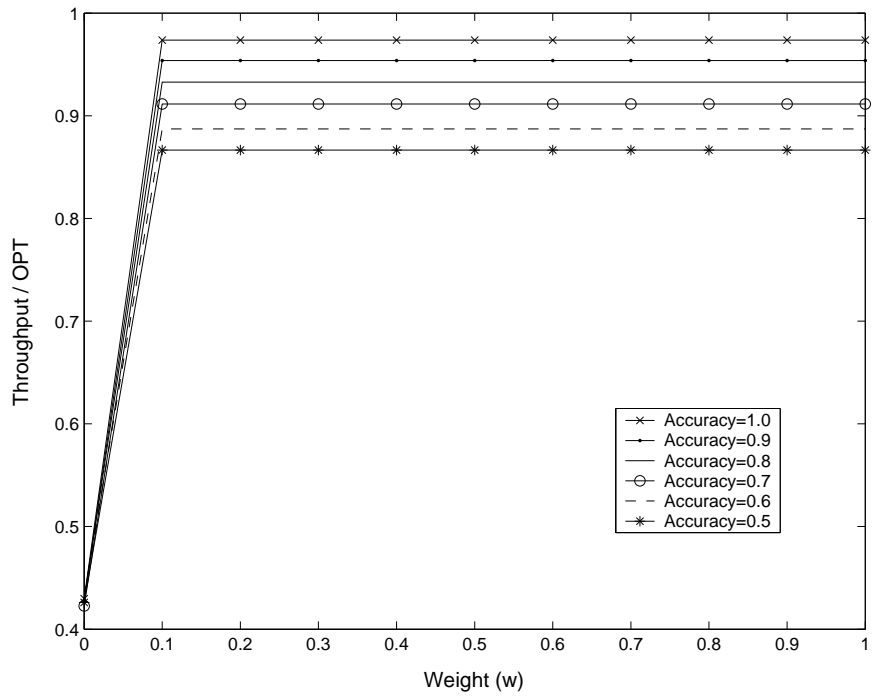

Fig. 2. Estimation Accuracy (p) vs. throughput for 1 user, 1 channel system with $\alpha=1$.

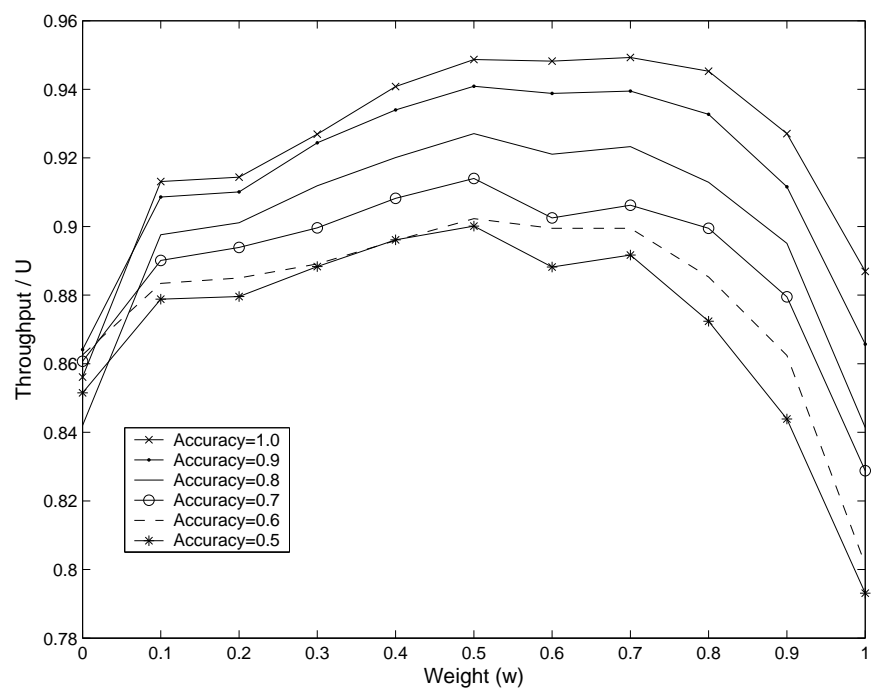

Fig. 3. Estimation Accuracy (p) vs. throughput for 4 user, 1 channel system with $\alpha=1$.

porate estimation errors and incorrect projections of the future transmission. The results suggest that a single look-ahead, even with inaccurate channel state prediction, may result in significant performance improvements over a zero lookahead algorithm. For a 1-user, 1-channel system, PMUMCPC can achieve throughput values of $86 \%$ and $97 \%$ of the optimal offline throughput with estimation accuracies of $50 \%$ and $100 \%$, respectively, whereas only $43 \%$ throughput is achievable without look-ahead. PMUMC-PC performs well for multi-user multi-channel systems as well, with a throughput of $95 \%$ with accurate estimates, and a throughput of $90 \%$ with $70 \%$ reliable estimates for a 20-user 5-channel system. Thus, most of the potential prediction gains can be obtained through a single look-ahead, and imperfect estimates. The 


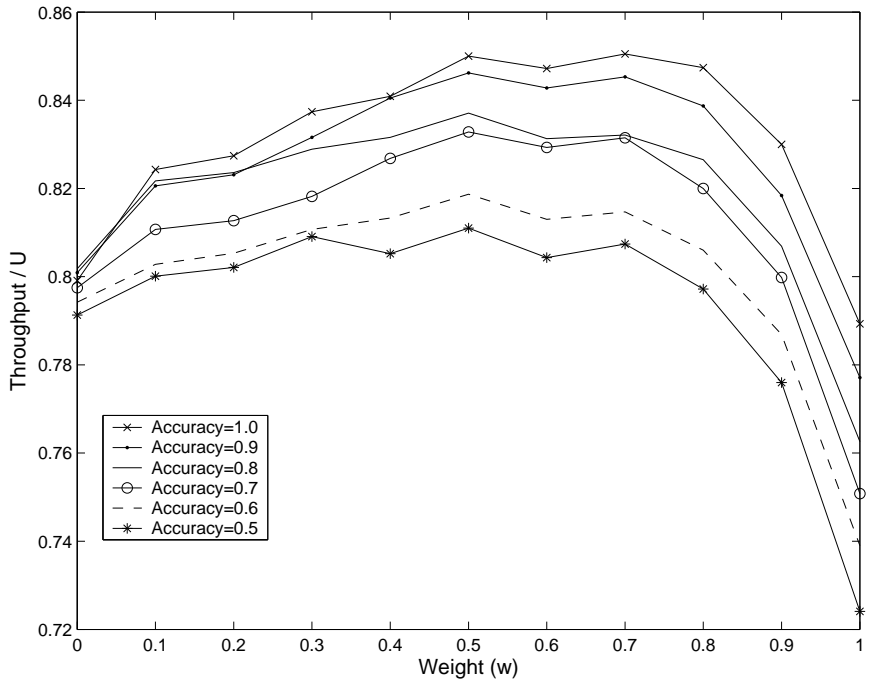

Fig. 4. Estimation Accuracy (p) vs. throughput for 5 user, 5 channel system with $\alpha=1$

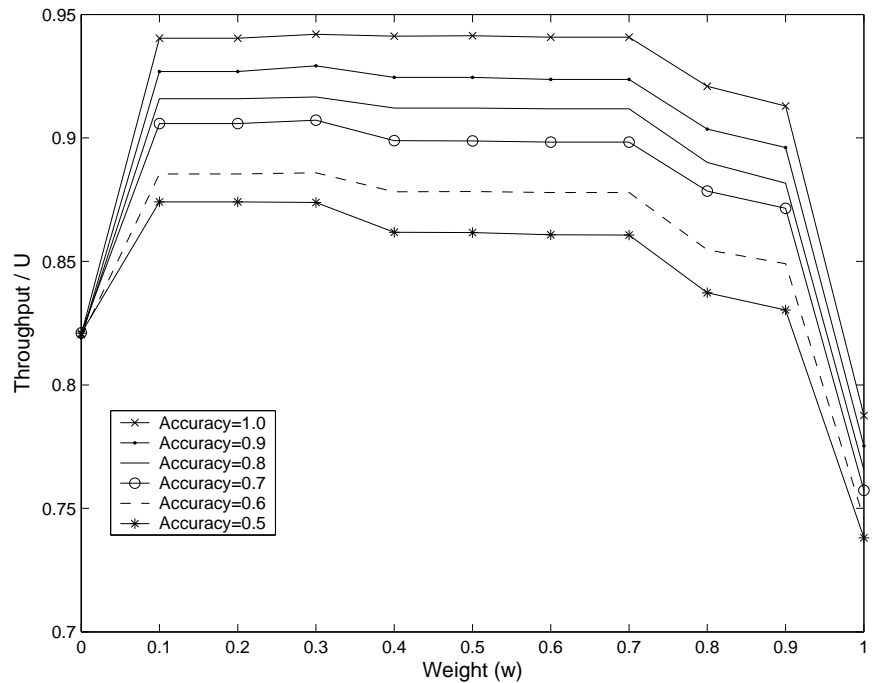

Fig. 5. Estimation Accuracy (p) vs. throughput for 20 user, 5 channel system with $\alpha=1$.

resource consumption for obtaining accurate estimates can be assessed based on these observations. We also observed that the presence multiple users favors a greedier approach that gives less weight to the future transmission projections.

A natural extension of this work would be an algorithmic complexity analysis of the problem [7], and developing QoSsensitive scheduling algorithms. Complementary techniques to reduce channel monitoring requirements, such as sub-carrier grouping where we have preliminary results [8], also constitute interesting research directions.

\section{REFERENCES}

[1] S. Catreux, V. Erceg, D. Gesbert, and R.W. Heath, "Adaptive modulation and MIMO coding for broadband wireless data networks," IEEE Communications Magazine, vol. 40, pp. 108-115, June 2002.

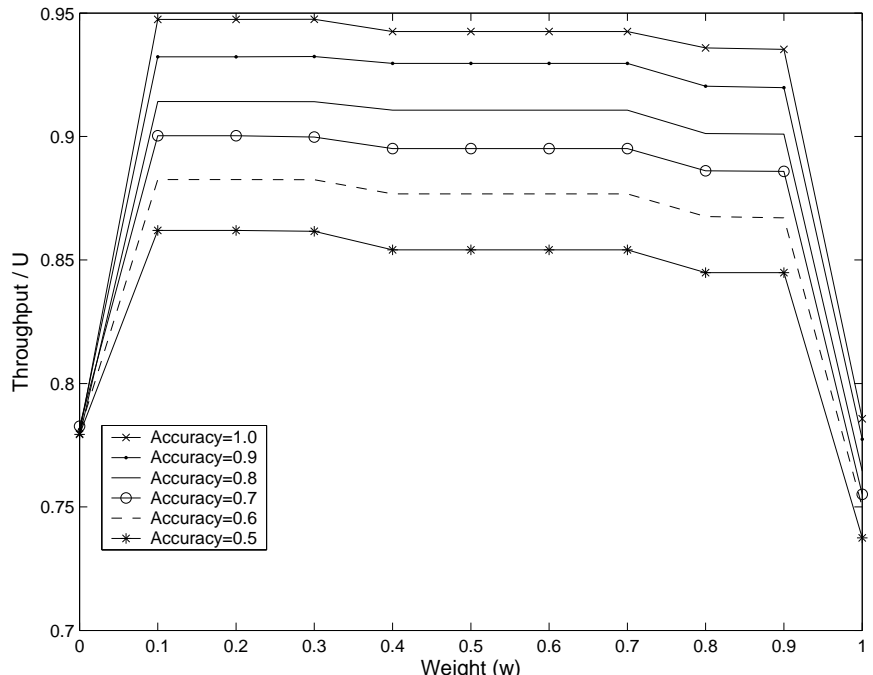

Fig. 6. Estimation Accuracy (p) vs. throughput for 40 user, 5 channel system with $\alpha=1$.

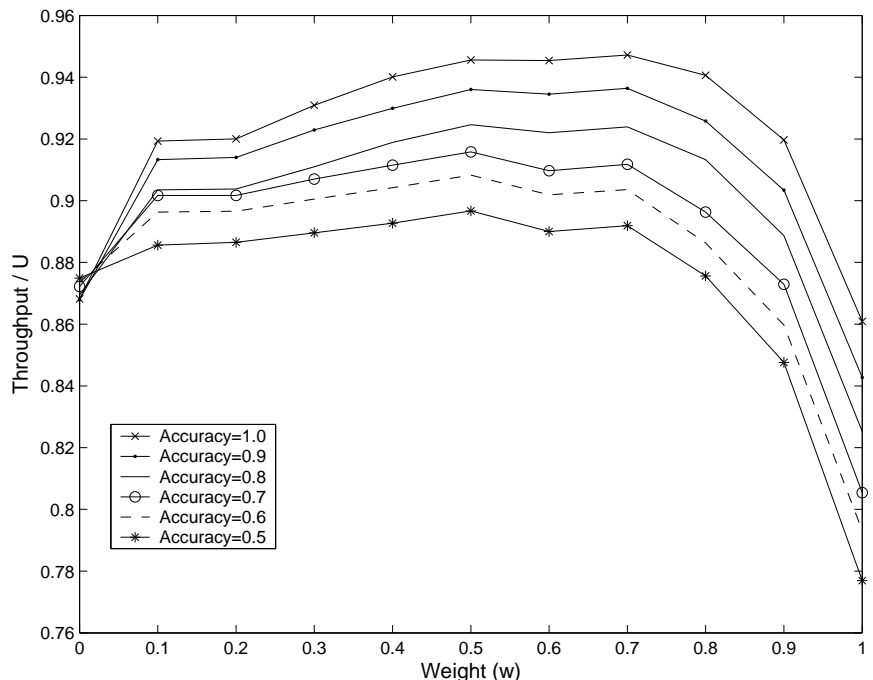

Fig. 7. Estimation Accuracy (p) vs. throughput for 5 user, 5 channel system with $\alpha=4$

[2] V. Tsibonis, L. Georgiadis, and L. Tassiulas, "Exploiting wireless channel state information for throughput maximization," in Proceedings of IEEE Infocom '03, April 2003, pp. 301-310.

[3] S. Kulkarni and C. Rosenberg, "Opportunistic scheduling policies for wireless systems with short term fairness constraints," in Proceedings of Globecom 2003, December 1992, pp. 533-537.

[4] X. Liu, E.K.P. Chong, and N. Shroff, "Opportunistic transmission scheduling with resource-sharing constraints in wireless networks," IEEE JSAC, vol. 19, no. 10 , pp. 2053-2064, Oct. 2001.

[5] T. Keller and L. Hanzo, "Adaptive multicarrier modulation: a convenient framework for time-frequency processing in wireless communications," Proceedings of the IEEE, vol. 88, pp. 1369-1394, August 1990.

[6] A. Arora and H.A. Choi, "Wait-dominate-hold," personal communication.

[7] A. Arora, F. Jin, G. Sahin, and H.A. Choi, "Throughput maximization in multi-carrier wireless networks with predictive link adaptation," July 2004 , in preparation.

[8] F. Jin, G. Sahin, A. Arora, and H.A. Choi, "The effects of the sub-carrier grouping on multi-carrier channel-aware scheduling," in Proceedings of Broadnets 04, October 2004, to appear. 